\title{
Reducing the uncertainty of strain gauge amplifier calibration
}

\author{
Miha Hiti \\ Slovenian National Building and Civil Engineering Institute (ZAG), Dimičeva 12, 1000 Ljubljana, Slovenija
}

\begin{abstract}
The article presents a method for calibration of strain gauge bridge amplifiers with improved uncertainty in low voltage ratio range. The procedure is based on combining traditional calibration of the amplifier at one point and linearity determination of the rest of the range. Traditional calibration is performed by a calibrated strain gauge bridge simulator at a reference value where measurement uncertainty is adequate, and the linearity is determined by a combinatorial calibration method with lower uncertainty, employing a special resistance circuit. Uncertainty in the lower part of the amplifier range can be significantly improved, resulting in a combined relative standard uncertainty below $2.5 \times 10^{-5}$ for the range from $0.04 \mathrm{mV} / \mathrm{V}$ to $2.5 \mathrm{mV} / \mathrm{V}$.
\end{abstract}

\section{Section: RESEARCH PAPER}

Keywords: calibration; strain gauge; simulator; amplifier; uncertainty

Citation: Miha Hiti, Reducing the uncertainty of strain gauge amplifier calibration, vol. 6, no. 4, article 11, December 2017, identifier: IMEKO-ACTA-06 (2017)$04-11$

Editor: Paolo Carbone, University of Perugia, Italy

Received June 19, 2016; In final form November 24, 2017; Published December 2017

Copyright: (C) 2017 IMEKO. This is an open-access article distributed under the terms of the Creative Commons Attribution 3.0 License, which permits unrestricted use, distribution, and reproduction in any medium, provided the original author and source are credited

Funding: The authors acknowledge the financial support from the Slovenian Research Agency (research core funding No. P2-0273)

Corresponding author: Miha Hiti, e-mail: miha.hiti@zag.si

\section{INTRODUCTION}

Strain gauge bridge transducers which are used for the measurement of mechanical quantities such as force, torque and pressure, need an instrument for excitation of the strain gauge bridge and for the measurement and indication of the bridge output signal, which depends on the load applied to the transducer. Strain gauge bridge measuring amplifiers usually perform these tasks. They indicate the result of the measurement as the ratio of the bridge supply voltage and the bridge output voltage. Figure 1 shows a typical transduceramplifier circuit in a 6-lead configuration.

As an important part of the measuring chain (or measuring system), bridge measuring amplifiers should be verified regularly. This is usually performed by calibration with strain gauge bridge simulators, which provide defined voltage ratio values as reference values.

Such simulators, in turn, need to be calibrated themselves for each reference value they output. For high precision simulators (e.g. $225 \mathrm{~Hz}$ carrier frequency type), the typical expanded calibration uncertainty $U$ of the $2 \mathrm{mV} / \mathrm{V}$ range is about $0.00001 \mathrm{mV} / \mathrm{V}$ at NMI level [1], [2] and $U=0.00002$ $\mathrm{mV} / \mathrm{V}$ at calibration laboratory level for further dissemination, leading to amplifier calibration uncertainties $U=0.00002 \mathrm{mV} / \mathrm{V}$ and above.

Such calibration uncertainties consecutively lead to large uncertainty of strain gauge amplifier measurements in the lower part of the range [3] when expressed in relative terms with respect to the measured value, as is typical for force and torque calibration. Figure 2 shows the relative standard uncertainty contribution of the simulator if the amplifier is calibrated with

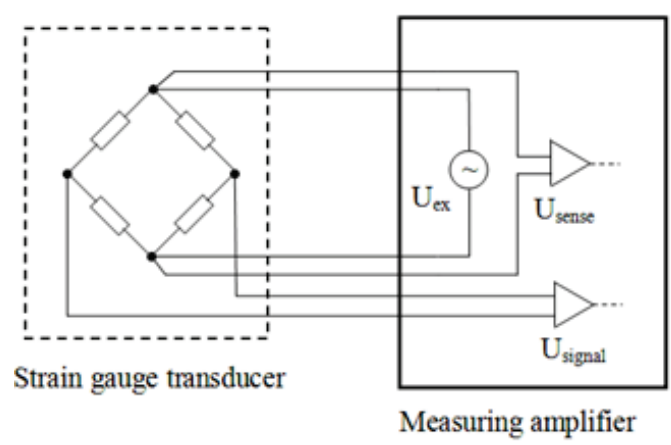

Figure 1. Strain gauge bridge transducer with 6-lead connection to the bridge amplifier in a force measuring system. 


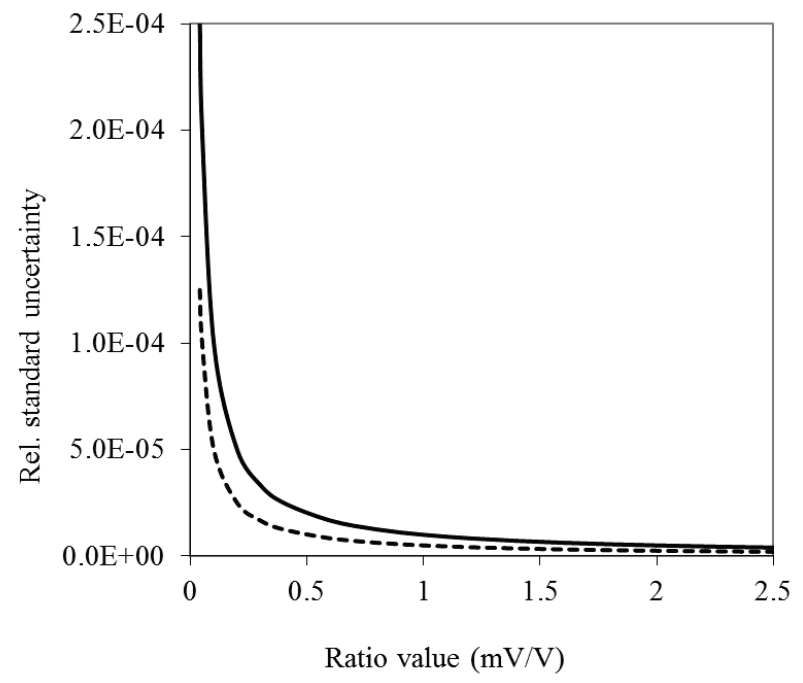

Figure 2. Relative standard uncertainty $w$ of a typical calibrated high precision strain gauge bridge simulator with $U=0.00002 \mathrm{mV} / \mathrm{V}$ (solid line) and the best available relative standard uncertainty at NMI level for $U=0.00001 \mathrm{mV} / \mathrm{V}$. (dotted line).

traditional calibrator units, such as HBM K3608 or HBM BN100A simulators. For $U=0.00002 \mathrm{mV} / \mathrm{V}$ (for $\mathrm{k}=2$ ) expanded calibration uncertainty and therefore $u=0.00001$ $\mathrm{mV} / \mathrm{V}$ standard uncertainty, the relative standard uncertainty $w$ ( $u$ divided by the measured value) at $2 \mathrm{mV} / \mathrm{V}$ is $5 \times 10^{-6}$, rising to $w=5 \times 10^{-5}$ at $0.2 \mathrm{mV} / \mathrm{V}$, and to $w=2.5 \times 10^{-4}$ at $0.04 \mathrm{mV} / \mathrm{V}$, which is the typical $2 \%$ low range limit of $2 \mathrm{mV} / \mathrm{V}$ nominal range force transducer. The best available relative standard uncertainty of simulator calibration at NMI level with $U=0.00001 \mathrm{mV} / \mathrm{V}$ expanded uncertainty is also shown, where the relative standard uncertainty $w$ reaches $1.25 \times 10^{-4}$ at 0.04 $\mathrm{mV} / \mathrm{V}$ ratio. Such uncertainties significantly exceed the available uncertainty of the realization of mechanical quantities, where, for example, force standard machines offer relative expanded uncertainties $W$ of $1 \times 10^{-5}$.

Calibrating the amplifier together with the transducer as a measuring system is one solution to overcome this limitation, but it limits the transducer to be always used with the same amplifier. The transducer calibration can be void in the case of amplifier replacement if the calibration data of amplifiers is not available.

If the transducer is used with another amplifier than the one employed originally during calibration, i.e. with a replacement amplifier, both amplifiers, original and replacement, should be calibrated to assure comparability of results. Furthermore, deviations and calibration uncertainties of both amplifiers should be considered [4], [5]. If both amplifiers are not checked with the same simulator, where the simulator is used as a comparator rather than a traceable reference, the uncertainty of the amplifier calibration should be evaluated and taken into account as necessary.

According to the international standard ISO 376 [4], replacing the amplifier (called simplified an indicator in the standard) is allowed if the following requirements are met:

Both amplifiers should be calibrated and traceable to national standards.

Both amplifiers should have the same working parameters (excitation voltage and frequency) and comparable resolution.
Calibration uncertainties of original and replacement amplifier should not significantly influence the total uncertainty of the force measuring chain. As a recommendation, the uncertainty of the replacement amplifier should not exceed $1 / 3$ of the uncertainty of the entire system.

Whenever the amplifier is replaced, the contribution of the amplifier calibration should be compared against the calibration uncertainty of the measuring system, for the whole calibrated range, as the calibration uncertainty of the simulator can become the major uncertainty contribution and can increase the total measuring system uncertainty.

In Section 2 we discuss the effect of the replacement amplifier on the total measuring system uncertainty. Further, in Section 3, we present a method and a circuit for determining the linearity of the amplifier without the need to first calibrate the circuit, thus allowing lower uncertainties. In Section 4 we show the results of the application of the method for linearity determination and calibration on a high precision measuring amplifier. Finally, conclusion is given in Section 5 .

\section{UNCERTAINTY CONTRIBUTION OF THE MEASURING AMPLIFIER}

When a calibrated transducer-amplifier measuring chain is separated, the traceability of the system needs to be reestablished via $\mathrm{mV} / \mathrm{V}$ voltage ratio standard. A schematics of the calibration of a measuring chain is shown in Figure $3 \mathrm{a}$ and necessary steps that are required for assuring exchangeability of measuring amplifiers via $\mathrm{mV} / \mathrm{V}$ traceability is shown in Figure $3 \mathrm{~b}$.

In the first case, the calibration is valid for the whole chain only, as the effects of transducer and amplifier are not individually known and therefore cannot be separated. For such calibration, it is not possible to simply replace the amplifier, as the original amplifier characteristics (e.g. linearity, deviation) are not known. While the calibration results are typically attributed to the transducer, they are actually only valid for the whole force measuring system, the transducer with original amplifier.

If the option of replacing the amplifier is required, then the calibration data for the transducer alone need to be provided, and not for the whole system. Thus, the effect of the amplifier

a) Calibration of measuring chain

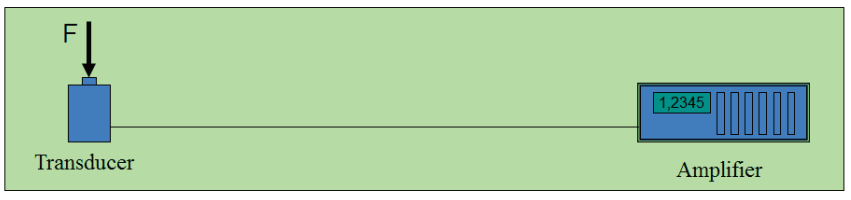

b) Separate calibration of tansducer and amplifier

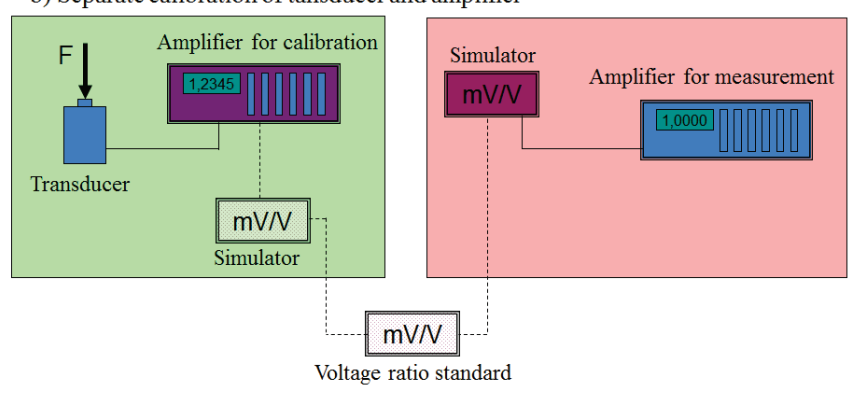

Figure 3. Comparison of (a) calibration of a measuring chain and (b) separate calibration of transducer and amplifier. 
should be eliminated from the measuring chain. For this, the amplifier must first be calibrated, to assess the amplifier characteristics, and then the influence of the amplifier should be considered - either the calibrated values of the system corrected for any amplifier deviation or the necessary corrections included as an additional uncertainty component. In any case, the uncertainty of the calibration of the amplifier will have to be included in the transducer calibration uncertainty.

Before the transducer can be used with a replacement amplifier, the replacement amplifier must also first be calibrated, and any deviations should be taken into account (corrected or included as additional uncertainty component). Then, the calibration uncertainty of the replacement amplifier needs be included in the uncertainty budget for the newly assembled measuring system.

To illustrate the scale of calibration uncertainty of the amplifier with respect to the uncertainty of the measuring chain (transducer with amplifier) an example is given in Figure 4, based on calibration data for a $100 \mathrm{kN}$ force transducer taken from an actual calibration certificate. The calibration uncertainty of the measuring chain is shown with a solid line, as a relative standard uncertainty. The force transducer in this example was calibrated in the range from $5 \mathrm{kN}$ to $100 \mathrm{kN}(5 \%$ to $100 \%$ of the nominal range) in a force standard machine with $0.002 \%$ expanded uncertainty. For comparison, the calibration uncertainty of the amplifier is shown with a dashed line, for a nominal output of the transducer of $2 \mathrm{mV} / \mathrm{V}$ at nominal load. The calibration uncertainty of the amplifier is specified in its calibration certificate as $0.00002 \mathrm{mV} / \mathrm{V}$ from 0.1 $\mathrm{mV} / \mathrm{V}$ to $2 \mathrm{mV} / \mathrm{V}$. It can be seen that, while the amplifier uncertainty is much lower than the measurement chain uncertainty for the upper range of the transducer, it significantly exceeds the measuring chain calibration uncertainty at lower force values. When replacing the amplifier, the uncertainty of both amplifiers should be taken into account, further increasing the effect of the amplifier calibration uncertainty.

As can be seen from this figure, the amplifier uncertainty exceeds $1 / 3$ of the transducer calibration uncertainty for values below $30 \%$ of the nominal range of the transducer. It results in a significant increase of the total uncertainty and should therefore not be neglected. This is also in agreement with the

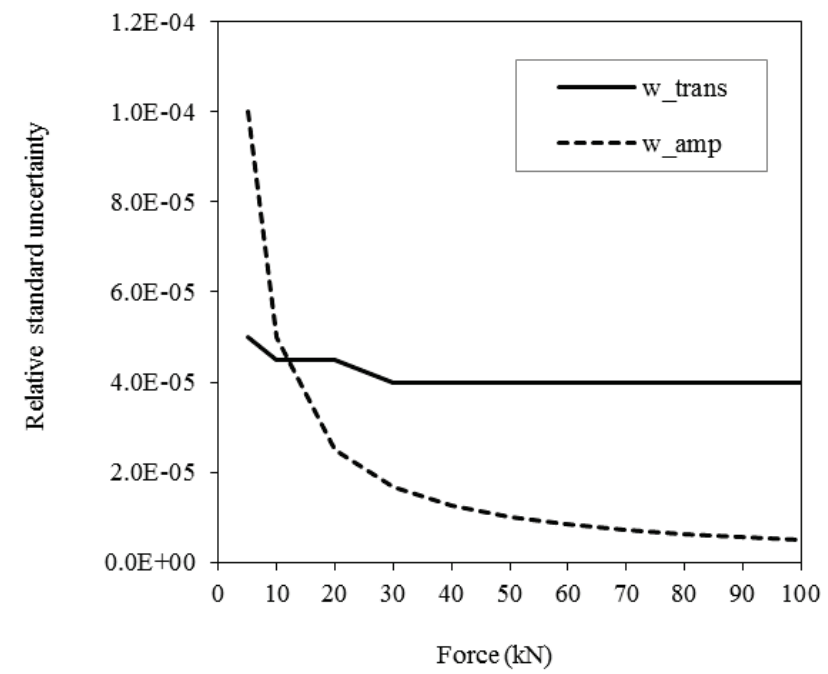

Figure 4. Comparison of relative standard uncertainty of calibration of a 100 $\mathrm{kN}$ force transducer chain ( $\mathrm{w}_{\text {trans }}$ ) and relative uncertainty of amplifier calibration $\left(w_{\text {amp }}\right)$. recommendation in ISO 376 regarding suitability of replacement amplifiers.

Figure 5 shows the expanded uncertainty $W_{\text {trans }}$ for the case where the $100 \mathrm{kN}$ force transducer is calibrated together with the amplifier as a measuring chain (solid line), and the resulting expanded uncertainty $W_{\text {trans+amp }}$ when a replacement amplifier is used after calibration (dashed line). For the second case, the combined standard uncertainty $w_{\text {transtamp }}$ is calculated from contributions of the original calibration uncertainty of the transducer chain $w_{\text {trans }}$ and additional calibration uncertainties of each amplifier (original amplifier - $w_{\text {amp_NMI }}$ and replacement amplifier - wamp_LAB) according to (1). In this example, both amplifiers have the same calibration uncertainty contribution (best available).

$W_{\text {trans }+ \text { amp }}=2 \cdot \sqrt{w_{\text {trans }^{2}}{ }^{2}+w_{\text {amp_NMI }^{2}}+w_{\text {amp_LAB }^{2}}}$.

The actual uncertainty contributions from amplifier calibration (absolute $U_{\text {amp }}$ and relative $W_{\text {amp }}$ ) and from transducer chain calibration ( $\left.W_{\text {trans }}\right)$ are shown in Table 1 . The output of the transducer ranges from $0.04 \mathrm{mV} / \mathrm{V}$ at $2 \%$ to 2 $\mathrm{mV} / \mathrm{V}$ at $100 \%$ nominal range. As the amplifier is not calibrated at $0.04 \mathrm{mV} / \mathrm{V}$, the uncertainty is estimated from the rest of the range. Also, calibration of the transducer is not available for $2 \mathrm{kN}$ force step, but is expected to be much less than the amplifier uncertainty.

To reduce the effect of the amplifier calibration uncertainty and to keep the low uncertainty of measuring system calibration also at the lower values of the range, an additional evaluation of

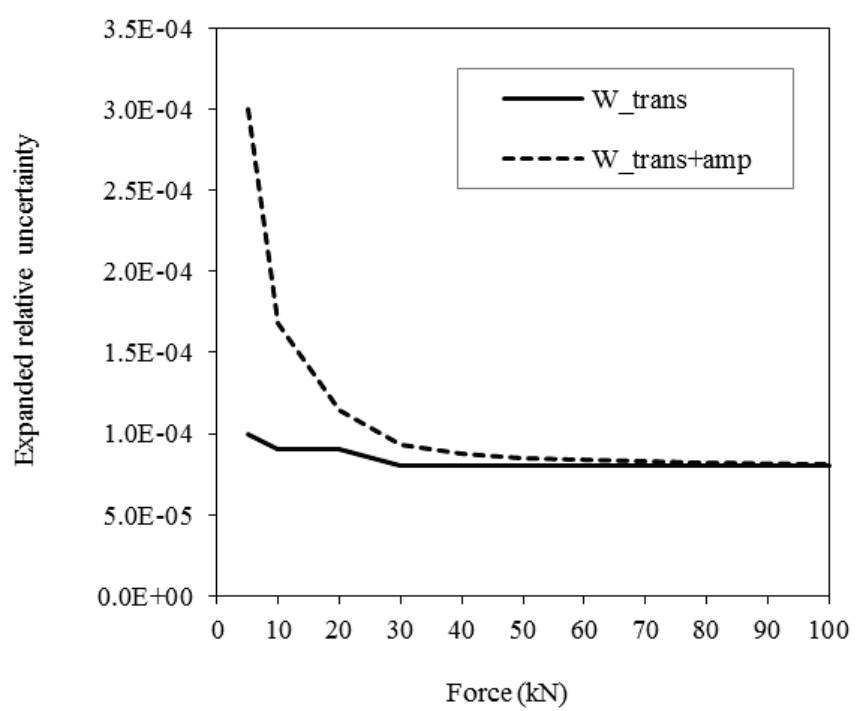

Figure 5. Comparison of expanded relative calibration uncertainties of a transducer chain $\left(W_{\text {trans }}\right)$ and of a calibration of a transducer with replacement amplifier ( $\left.W_{\text {trans+amp }}\right)$.

Table 1. Comparison of uncertainty contributions.

\begin{tabular}{ccccc}
\hline Range & $\begin{array}{c}\text { Output } \\
{[\mathrm{mV} / \mathrm{V}]}\end{array}$ & $\begin{array}{c}\mathbf{U}_{\mathrm{amp}} \\
{[\mathrm{mV} / \mathrm{V}]}\end{array}$ & $\begin{array}{c}\mathbf{W}_{\text {amp }} \\
{[\%]}\end{array}$ & $\begin{array}{c}\mathbf{W}_{\text {trans }} \\
{[\%]}\end{array}$ \\
\hline $2 \%$ & 0.04 & $0.00002\left(^{*}\right)$ & 0.050 & $/$ \\
$5 \%$ & 0.1 & 0.00002 & 0.020 & 0.010 \\
$10 \%$ & 0.2 & 0.00002 & 0.010 & 0.009 \\
$20 \%$ & 0.4 & 0.00002 & 0.005 & 0.009 \\
$50 \%$ & 1.0 & 0.00002 & 0.002 & 0.008 \\
$100 \%$ & 2.0 & 0.00002 & 0.001 & 0.008 \\
\hline
\end{tabular}

$(*)$ estimate 
the amplifier can be performed by determining the linearity of the amplifier (deviation from a straight line response) with low uncertainty. By calibrating the amplifier with a calibrated simulator at one reference value - at higher ratio values, where relative uncertainty is adequate - and using an alternative linearity check method with low uncertainty to verify the rest of the range, the measurement uncertainty in the lower range of the measuring system can be improved.

\section{MEASURING EQUIPMENT AND PROCEDURE}

\subsection{Combinatorial calibration method}

To solve the problem of evaluation of measuring instruments, where the calibration uncertainty of reference standards is too high, a calibration method that does not require traceably calibrated equipment can be applied [6]. The method, also called combinatorial method, has been in the last 20 years successfully applied in many different fields, e.g. thermometry bridge calibration [7].

The combinatorial calibration method is based on measuring a set of artefacts: each individual artefact separately and also all possible combinations of these artefacts. From the deviation of measured values of combinations of artefacts and calculated results from the same combinations, the non-linearity of the system can be estimated. Additionally, if one artefact is calibrated, a full calibration can also be performed.

\subsection{Circuit for combinatorial method}

To apply the combinatorial method to evaluation of measuring amplifiers, a suitable set of artefacts in necessary. A circuit fulfilling the requirements, which can also act as a strain gauge bridge simulator, is presented in [8]. A general schematics of the circuit employed for linearity determination is shown in Figure 6. The circuit is based on a variable resistive voltage divider comprised of base resistors, with additional resistor networks connected to the output leads of the divider to reduce the variation of the output resistance of the circuit. The output ratio is varied by tapping the output over a single base resistor or a combination of consecutive base resistors.

In the case of the circuit in Figure 6, the set of artefacts is defined by the four base resistors, which form a part of the voltage divider network. Extending the circuit to eight base resistors allows measurements of 36 non-zero output combinations, from which the estimation of the linearity error can be calculated. The output ratios of the divider, as well as input and output resistance of the circuit, can be adapted to

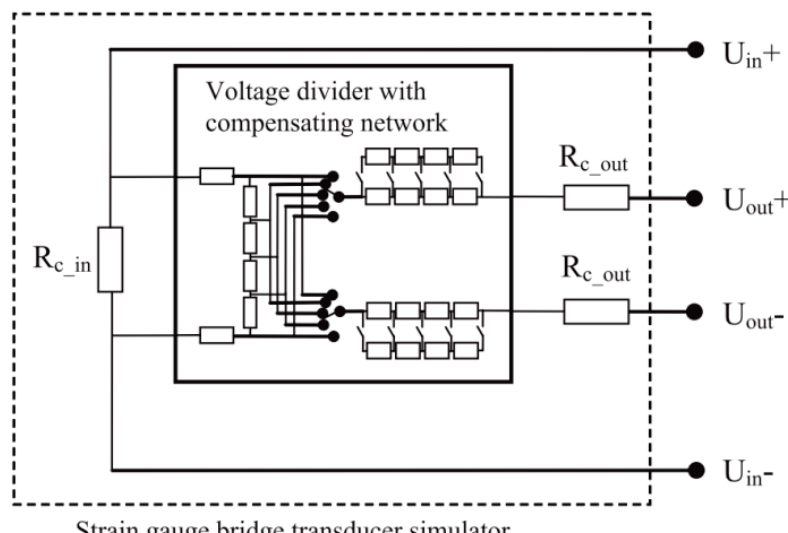

Figure 6. Schematics of a resistive voltage divider circuit for application of the combinatorial method for amplifier linearity determination. meet the requirements. The actual circuit built to check the linearity of the amplifier was designed for a $350 \Omega$ input and output resistance and a $2.5 \mathrm{mV} / \mathrm{V}$ nominal output ratio. With appropriately selected values for the eight base resistors, it can cover the range from about $0.04 \mathrm{mV} / \mathrm{V}$ to $2.5 \mathrm{mV} / \mathrm{V}$.

\subsection{Linearity determination}

Even without the calibration of the resistors, the circuit can be applied for the determination of linearity of measuring amplifiers. The circuit is connected to the amplifier in place of the transducer and the indication on the amplifier is recorded for each available output combination. When ratio values for all combinations have been measured, deviations of the measured sum of selected resistors and the calculated sum of selected resistors are determined. As the errors for base resistors are not known, they are estimated by a least square fit, based on the error distribution from all measurements. A correction function is calculated for the resulting deviation values which represents the nonlinearity, and residuals of the fit serve as standard uncertainty estimation of the linearity measurement.

When the circuit is applied in combination with the combinatorial calibration method, the resulting uncertainty of the linearity determination depends mainly on the quality of the measuring instrument. If it is applied to high precision amplifiers [9], it is possible to reach a standard uncertainty of the linearity determination in the range of $0.000002 \mathrm{mV} / \mathrm{V}$ or better [10].

\subsection{Reference point calibration}

The result of the linearity determination alone is not enough to calibrate the amplifier. Additional traceable calibration must be performed at least at one non-zero ratio value to establish the absolute linear error of the amplifier indication. As the zero point is usually fixed by definition by setting the indication to zero at the beginning of the measurement or later subtracting the indication at zero load, only one calibration point is necessary to define the slope, as the line is expected to go through zero. The uncertainty of the calibrated absolute value defines the uncertainty of the slope - the linear error, and it scales in proportion to the measured value [6], [11]. For lower values it is proportionally lower and for higher values it is proportionally higher than at the reference point.

It is beneficial to choose the largest possible absolute calibration point, as its relative uncertainty contribution will be constant and attributed to the whole range, and therefore provide the lowest uncertainty in the lower part of the range, Figure 7. For the purpose of absolute calibration, traditional

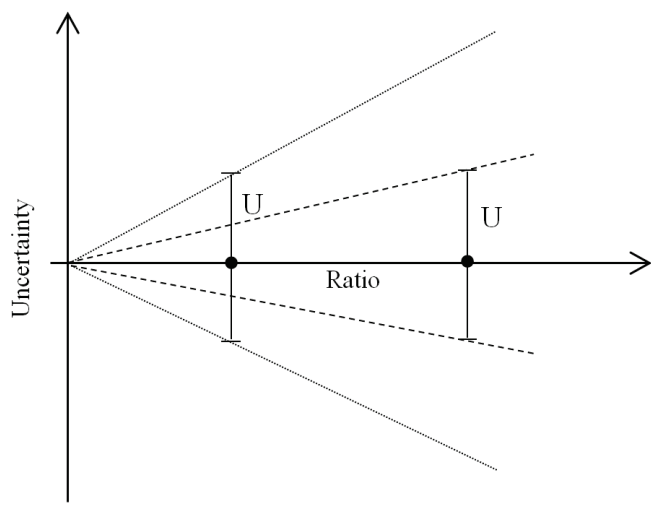

Figure 7. The uncertainty contribution of the absolute calibration point with respect to the selected calibration value. 
simulators can be employed as, depending on the selected range, the simulator relative calibration uncertainty can be adequate for the required task.

With zero value set, known linearity correction and its uncertainty, and one absolute calibration point for the slope, the instrument can be fully calibrated. At the expense of increased uncertainty contribution, the calibration range can also be extended above the calibrated point.

\section{RESULTS}

The evaluation was performed on an HBM DMP41 highprecision $225 \mathrm{~Hz}$ carrier frequency amplifier. The selected parameters were $2.5 \mathrm{mV} / \mathrm{V}$ range, $5 \mathrm{~V}$ excitation voltage and $0.1 \mathrm{~Hz}$ Bessel filter. The expanded calibration uncertainty of the amplifier at $2 \mathrm{mV} / \mathrm{V}$ using a traditional simulator was $U=0.00002 \mathrm{mV} / \mathrm{V}$, or $W=1 \times 10^{-5}$ relative uncertainty. In combination with the linearity determination, the calibration of the amplifier in the whole range from $0.04 \mathrm{mV} / \mathrm{V}$ to $2.5 \mathrm{mV} / \mathrm{V}$ was performed.

Figure 8 shows the result of the linearity determination with the combinatorial calibration method. The figure shows the resulting deviation of measurement of 36 possible resistor combinations (black dots) and the fit of the linearity error (solid curve) with its estimated standard uncertainty. The calculated standard deviation of the residual errors is about 0.000001 $\mathrm{mV} / \mathrm{V}$, shown with solid error bars. The linearity check with a calibrated simulator HBM BN100A is also shown (dashed line with square markers) and the standard uncertainty of the linearity determination of the amplifier performed with the simulator is represented with dashed uncertainty bars. For the comparison, the resulting curves are referenced at $0 \mathrm{mV} / \mathrm{V}$ and $2 \mathrm{mV} / \mathrm{V}$. It can be seen that, while both measurements are in good agreement, the uncertainty of the linearity determination with the combinatorial method produces a much lower uncertainty than the linearity measurement with a calibrated simulator.

The results of the combinatorial method are sufficient to characterise the nonlinearity, but they do not provide enough information for the calibration of the amplifier, since the linear

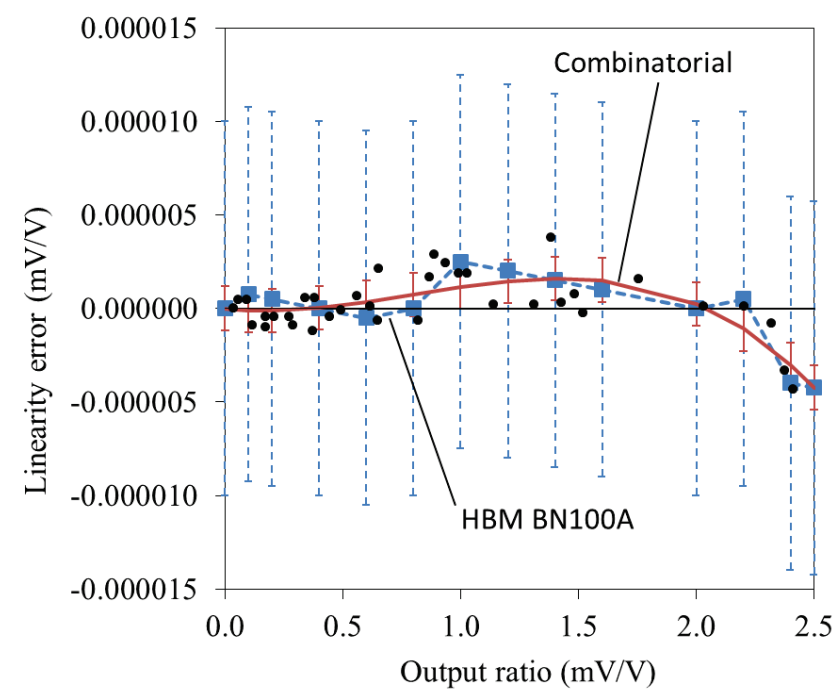

Figure 8. Amplifier linearity error determination with the combinatorial method (solid error bars) vs. calibration with a simulator (dashed error bars), referenced at $0 \mathrm{mV} / \mathrm{V}$ and $2 \mathrm{mV} / \mathrm{V}$. Error bars represent standard uncertainty. error is not known. An additional measurement at one ratio value is required to fully calibrate the instrument. If the absolute calibration at one point is made with $U=0.00002 \mathrm{mV} / \mathrm{V}$ expanded uncertainty, it will define the minimum uncertainty at that point. Together with the linearity determination uncertainty, the total calibration uncertainty of the instrument can be calculated.

Figure 9 shows the standard uncertainty contributions for the combination of absolute calibration of the amplifier at 2 $\mathrm{mV} / \mathrm{V}$ and linearity determination based on data from Figure 8 . The dashed line represents the reference point calibration standard uncertainty of $u=0.00001 \mathrm{mV} / \mathrm{V}$. For the linear instrument error, this value can be scaled proportionally with the ratio value, its relative contribution remaining constant (dotted line) - the contribution due to calibration uncertainty. The second contribution is the standard uncertainty of the linearity determination (thin solid line) performed with the resistor circuit and combinatorial method. The final combined standard uncertainty is shown by the thick solid line. The combined uncertainty is calculated according to (2), where $u_{\mathrm{c}}$ is the combined standard uncertainty, $u_{\text {sim_prop }}$ the proportional part of the standard uncertainty contribution of the absolute point calibration, and $u_{\text {lin_check }}$ is the standard uncertainty contribution of the linearity determination employing the combinatorial calibration method:

$u_{\mathrm{c}}=\sqrt{u_{\text {sim_prop }}^{2}+u_{\text {lin_check }}^{2}}$.

It can be seen in Figure 9 that the dominant uncertainty contribution for most of the range is the calibration uncertainty arising from the simulator calibration uncertainty. Compared to a calibration employing only the simulator, the achieved uncertainty with the proposed procedure has been reduced significantly in the lower range of ratio values, and slightly increased for ratio values above the absolute calibration point.

Figure 10 shows the final calibration result of the amplifier using the combinatorial calibration method and one reference point calibration with a calibrated simulator. The result of the linearity determination from Figure 8 is now combined with the established deviation during the amplifier calibration at 2 $\mathrm{mV} / \mathrm{V}$. The uncertainty of the linearity determination is enlarged by the proportional part of the reference point

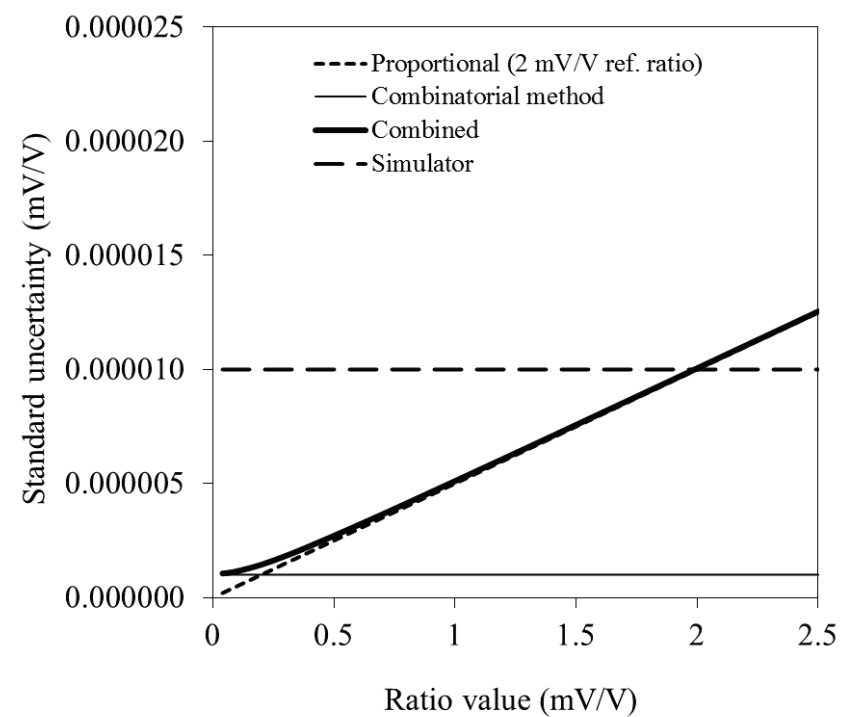

Figure 9. Combined standard uncertainty of absolute calibration at $2 \mathrm{mV} / \mathrm{V}$ $(\mathrm{U}=0.00002 \mathrm{mV} / \mathrm{V})$ and linearity determination for the whole range. 


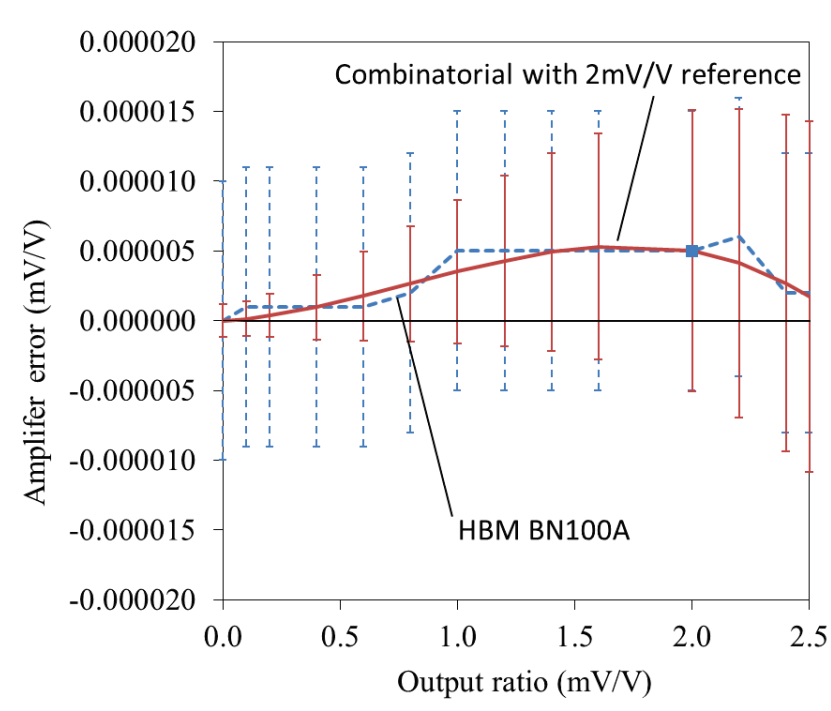

Figure 10. Full calibration of the amplifier with absolute calibration at 2 $\mathrm{mV} / \mathrm{V}$ and determined linearity for the whole range (solid error bars). Result of the traditional calibration is also shown (dashed error bars). Both curves are expected to go through zero as initially the indication is set to zero. Error bars are for standard uncertainty.

\section{calibration uncertainty.}

In Figure 11 the same measurement example from Figure 9 is shown, but standard uncertainty contributions are expressed as relative standard uncertainties. We can see that the relative calibration uncertainty of the simulator increases significantly for lower ratio values (dashed line). The proportional part of the absolute calibration uncertainty taken for the $2 \mathrm{mV} / \mathrm{V}$, when expressed as relative uncertainty, is constant for the whole calibration range (dotted line). The contribution of the linearity determination expressed as relative contribution is shown by the thin solid line. The combined relative standard uncertainty $w$ is below $1 \times 10^{-5}$ for the most of the range and below $2.5 \times 10^{-5}$ for the whole evaluated range. Again, the

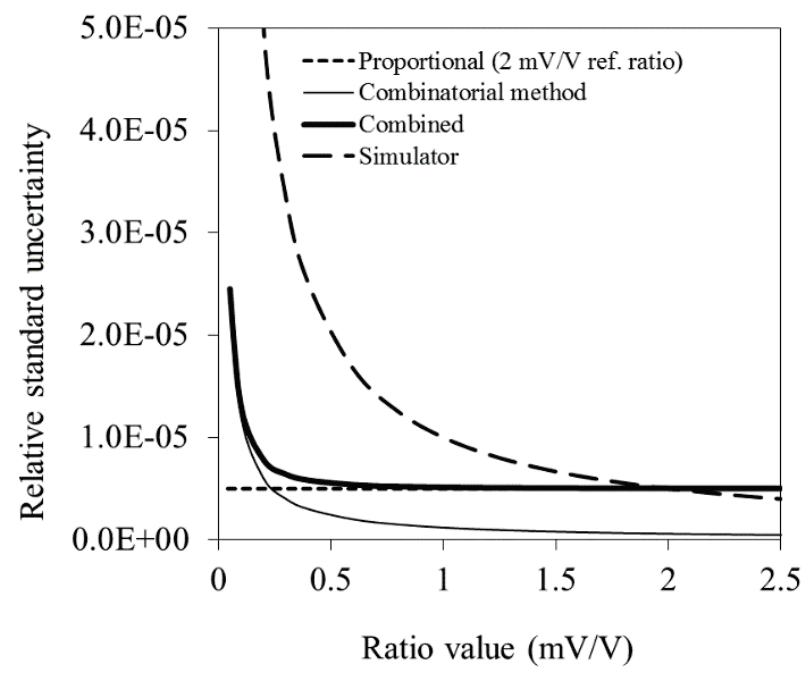

Figure 11. Combined standard uncertainty $w$ of absolute calibration at 2 $\mathrm{mV} / \mathrm{V}\left(U=0.00002 \mathrm{mV} / \mathrm{V}\right.$ or $\left.W_{(2 \mathrm{mV} / \mathrm{v})}=5 \times 10^{-6}\right)$ and uncertainty of the linearity determination for the whole range. reduced uncertainty compared to employing only the simulator is evident at low ratio values. Above the $2 \mathrm{mV} / \mathrm{V}$ absolute reference point, the uncertainty has been slightly increased.

In this paper, only the calibration uncertainty of the amplifier calibration at one value and the uncertainty of the linearity check are considered. Other contributions, such as resolution of the instrument, drift of the simulator ratio value and other possible contributions are not explicitly taken into account.

\section{CONCLUSIONS}

The results of the evaluation show improvement in calibration uncertainty of measuring amplifiers in comparison to traditional strain gauge simulator calibration only. Combining the traditional simulator based calibration and combinatorial calibration based linearity determination, improved calibration in the lower part of the range can be achieved. With the presented method, the relative standard uncertainty $w$ at 0.04 $\mathrm{mV} / \mathrm{V}$ could be reduced from typical values of $2.5 \times 10^{-4}$ to values below $2.5 \times 10^{-5}$.

The improved calibration uncertainty allows separate calibration of transducers and amplifiers and thus interchangeable transducer-amplifier combinations, while preserving acceptable calibration uncertainty levels for most scientific and industrial applications.

\section{REFERENCES}

[1] R. Vollmert, G. Ramm, "Realization, maintenance and dissemination of the measurand " $\mathrm{AC}$ voltage ratio in $\mathrm{mV} / \mathrm{V}$ " for strain gauge measurements", $18^{\text {th }}$ IMEKO Conference on Force, Mass and Torque 2002, Celle, Germany, 2002, pp. 145-150.

[2] Calibration and Measurement Capabilities - CMCs, The BIPM key comparison database, http:// kcdb.bipm.org, BIPM.

[3] D. Schwind and T. Hahn, "Investigation of the influence of carrier frequency or direct current voltage in force calibrations", XIX IMEKO World congress, Lisbon, Portugal, 2009, pp. 201204.

[4] ISO 376:2011; Metallic materials - Calibration of force proving instruments used for the verification of uniaxial testing machines

[5] Calibration guide EURAMET cg-4 version 2.0(03/2011): Uncertainty of force measurements, EURAMET, 2011.

[6] D.R. White, M.T. Clarkson, P. Saunders and H.W. Yoon "A general technique for calibrating indicating instruments", Metrologia, 45, 2008, pp. 199-210.

[7] D.R. White, "A method for calibrating resistance thermometry bridges", Procedings of TEMPMEKO '96, Torino, Italy, 1997, pp.129-134.

[8] M. Hiti, "Resistor network for linearity check of voltage ratio meters by combinatorial technique", Meas. Sci Technol.,Vol 26, No. 5, 2015, pp. 1-7.

[9] M.M. Schäck, "High-precision measurement of strain gauge transducers at the physical limit without any calibration interruption", IMEKO $22^{\text {nd }}$ TC3, $12^{\text {th }}$ TC5 and $3^{\text {rd }}$ TC22 International Conferences, Cape Town, Republic of South Africa, 2014, pp. 118-123.

[10] M. Hiti, "Alternative calibration procedure for strain gauge amplifiers”, XXI IMEKO World Congress 2015, Prague, Czech Republic, 2015.

[11] M. Borys, R. Schwartz, A. Reichmuth, R. Nater, "Fundamentals of Mass Determination" Springer-Verlag Berlin Heidelberg, 2012, ISBN 978-3-642-11937-8. 